$\begin{array}{ll}\text { Italique } & \text { Italique } \\ \text { Poésie italienne de la Renaissance }\end{array}$

XV $\mid 2012$

Varia

\title{
Veronica Franco et la dignité d'une courtisane
}

\section{Piotr Salwa}

\section{OpenEdition}

Journals

Édition électronique

URL : http://journals.openedition.org/italique/362

DOI : $10.4000 /$ italique.362

ISSN : 1663-4438

\section{Éditeur}

Librairie Droz

\section{Édition imprimée}

Date de publication : 1 décembre 2012

Pagination : 235-259

ISBN : 978-2-600-01639-1

ISSN : 1423-3983

\section{Référence électronique}

Piotr Salwa, «Veronica Franco et la dignité d'une courtisane », Italique [En ligne], XV | 2012, mis en ligne le 01 décembre 2015, consulté le 10 décembre 2020. URL : http://journals.openedition.org/ italique/362 ; DOI : https://doi.org/10.4000/italique.362 


$$
\text { Piotr S S LWA }
$$

VER O N I C A F R A N C O

E T L A D I G N T É D' U NE C O UR T IS A N E 



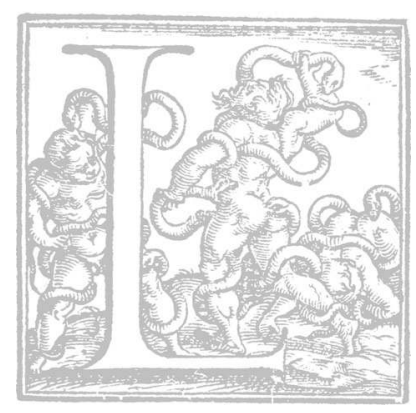

'bistoire littéraire «mineure 》 du XVT siècle offre parfois des rencontres et des coïncidences inattendues et surprenantes, qui par hasard mettent en scène des personnages exceptionnels. Dans une de ces occasions, la poésie italienne s'est présentée sur le chemin qui unissait la Pologne et la France.

En juin I574, Henri de Valois, futur roi de France et alors roi de Pologne, arrivait à Venise. Il avait accédé au trône de Cracovie en mai I573, après de longues mancuures diplomatiques, en tant que premier roi électif; après la mort du dernier souverain Jagellon la Pologne cessait d'être un royaume héréditaire et devenait une république aristocratique, dont le souverain était choisi par la noblesse, le plus souvent parmi les princes étrangers. En acceptant le trône polonais, le prince français avait dîu signer les pacta conventa et les articles nommés en référence à lui «du Roi Henri», des documents qui limitaient sérieusement le pouvoir royal et qui garantissaient aux nobles des libertés exceptionnelles à l'époque, la liberté de confession et la possibilité de refuser l'obéissance au roi, des conditions qui devaient probablement être difficiles à accepter par un souverain d'origine française. En fait, le jeune prince français et la Pologne ne se sont jamais aimés. Le premier trouvait son nouvel État polono-lituanien peu amical, caractérisé par un climat impossible, une pauvreté effrayante et des coutumes rudes et barbares; la cour de son côté trouvait le nouveau souverain difficile à assimiler, efféminé, suspect, étranger à tous points de vue. Le roi, que les nobles polonais avaient préféré au candidat Habsbourg, rejoignit son royaume en janvier I574 et il fut couronné en février de la même année. Mais son règne était destiné à durer très peu de temps. En juin I574, quand la nouvelle de la mort inattendue de son frère Charles IX parvint à Cracovie, Henri n'bésita pas à faire rapidement son choix, à quitter la capitale polonaise et se rendre à Paris pour ne pas perdre l'occasion de devenir roi de France. Il commit une erreur, après tant d'autres, dans ses relations avec les Polonais: il se mit en voyage sans avertir les dignitaires et les responsables de l'État. Le départ clandestin d'un souverain mal aimé et mal compris fut considéré comme une fuite. On se mit à sa poursuite pour lui faire changer d'avis, en vain. Le départ du roi provoqua une grave crise constitutionnelle: la 
Diète fit savoir au souverain qu'il perdait son trône polonais s'il ne revenait pas avant le mois de mai 1575 . Henri de Valois ne revint pas. Il n'est pas surprenant qu'il ne put, par la suite, gagner la sympathie, ni même la comprébension de l'bistoriographie polonaise.

Arrivé à Venise le I 8 juillet I574, Henri de Valois fut reçu avec les plus grands honneurs. Les descriptions des somptueuses cérémonies qui accompagnèrent son séjour dans la Serenissima évoquent dix journées de fêtes continuelles, à l'organisation desquelles les autorités vénitiennes engagèrent les artistes les plus renommés. ${ }^{\text {I }}$ Un grand tableau d'Andrea Vicentino représentant Henri III roi de France et de Pologne, accompagné par le doge Alvise I ${ }^{\text {er }}$ Mocenigo, et accueilli par le patriarche Giovanni Trevisan à San Nicolò di Lido orne encore aujourd'bui la Salle des Quatre Portes du Palais des Doges et il nous montre Henri et les autorités vénitiennes sur le pont construit par Andrea Palladio expressément pour cette occasion, avec un arc de triomphe et une loggia couverte de peintures par Veronèse et Le Tintoret. On y remarque également un grand nombre de bateaux, - les relations parlent de I20 à 200 embarcations qui accompagnaient l'illustre visiteur-, dont le célèbre Bucintoro.

C'est à l'occasion de cette visite que le prince rencontre Veronica Franco. Il avait alors 23 ans, et malgré les rumeurs sur ses penchants homosexuels, les autorités vénitiennes lui avaient offert la compagnie de cette cortigiana onesta d'une certaine réputation. On ne connaît pas les raisons pour lesquelles le choix s'était porté précisément sur elle, ni quel était exactement le statut des courtisanes auxquelles on attribuait la qualité d'honnêtes. Il est certain que Veronica Franco savait bien veiller à ses intérêts, en assurant ses « relations publiques 》 et en sollicitant la curiosité des étrangers de passage, qui n'étaient pas nécessairement intéressés par son activité de courtisane, à en croire la brève mention que fit Montaigne dans son Voyage en Italie. ${ }^{2}$ Quelqu'un a même qualifié Véronica, avec une désinvolture quelque peu excessive, comme une "major tourist attraction ». Mais il est certain que le roi passa chez elle une nuit et qu'il repartit en emportant avec lui un portrait d'elle, probablement une cuvre du Tintoret. De son côté, Veronica lui dédia une lettre et deux sonnets, qui n'étaient pas ses premiers essais poétiques.

Qui était cette Veronica Franco? Ce personnage, assez célèbre au XVI siècle avant d'être oublié pendant les siècles qui suivirent, a été 
récemment rappelé à l'attention du grand public par l'excellente biographie de Margaret Rosenthal, The Honest Courtesan, et le film qui en a été tiré, Dangerous Beauty. ${ }^{3}$ Dans une recension on peut lire: "La vie de Veronica Franco est vraiment fascinante. Sans argent et sans famille importante, elle a su profiter de son intelligence et de son talent, ainsi que de sa personnalité brillante, de sa beauté physique et de ses talents érotiques. L'ayant emporté sur de puissants adversaires dans le domaine juridique et littéraire, elle gagna la célébrité comme poétesse et comme écrivain, et elle fut admise à participer à d'importants événements politiques et littéraires de son temps. Ce personnage historique mérite toujours notre attention et notre admiration, plus de quatre cents ans après sa mort $\gg .^{4}$

Appartenant à la classe des cittadini originari de Venise, intermédiaire entre la noblesse et le peuple, elle était la fille d'une autre courtisane honnête, qui lui avait enseigné son métier. Très jeune fille, elle avait été mariée à un riche médecin, mais ce mariage tourna court, après une liaison extraconjugale et la naissance d'un enfant illégitime. Veronica dut alors se trouver une activité pour assurer sa vie. Elle avait probablement reçu une bonne éducation, dont on ne sait rien, qui servit de support à ses talents et à son intelligence.

En I575, elle publia un volume de Terze rime, et en Is80 un volume de Lettere familiari a diversi,' qui lui ouvrirent la porte des prestigieux milieux intellectuels et artistiques de la Sérénissime; elle participa aux débats et collabora à l'édition de ces anthologies littéraires très à la mode en Italie dans la deuxième moitié du siècle. ${ }^{6}$ Elle pouvait compter sur des amitiés, des connaissances et des protections parmi les citoyens les plus influents de Venise.

On sait aussi qu'elle fut contrainte de quitter la cité en I575 à cause de la peste, et qu'elle perdit à cette occasion une partie des biens qu'elle avait accumulés au cours des années de prospérité, quand sa maison avait été pillée. En I577, elle fut accusée de pratiques magiques devant l'Inquisition, mais elle sut se défendre et fut acquittée, probablement avec l'aide de ses amis haut placés. Les chercheurs qui ont examiné les actes du procès soulignent toutefois la fermeté des propos tenus par Veronica Franco et son comportement respectable et sérieux. À part cela, on ne sait pas beaucoup plus de sa vie: elle eut plusieurs enfants, elle était engagée dans les cuvres de charité, elle s'est rendue à Rome en pèlerinage, et elle eut l'intention de fonder un hospice pour les anciennes 
prostituées. Elle a probablement dû finir ses jours dans des conditions assez modestes, peut-être même dans la pauvreté, comme le suggère le lieu de sa dernière résidence vénitienne.

Pendant son fastueux séjour à Venise, Henri de Valois avait rencontré Veronica Franco, qui lui était offerte en tant que « cadeau 》 d'une nuit destiné à un allié politique d'importance; peut-être les autorités vénitiennes avaient-elles même cherché à confier à la courtisane quelque mission délicate, comme à un « agent de renseignement». Toutefois, le roi tomba sur une personnalité rebelle, qui était loin d'être satisfaite de son rôle ou de son statut social subalterne, et qui était loin de se résigner à la condition dans laquelle elle était communément considérée. Ses ambitions en effet étaient bien différentes.

Dans une de ses Lettere familiari, Veronica Franco exprime son opinion à propos du métier qu'elle exerce, et elle fait allusion à une situation analogue à celle qui était autrefois la sienne. En s'adressant à une mère qui pense à diriger sa fille vers la prostitution, elle écrit:

la rovina di lei non può essere separata dalla vostra et perché sete madre et perché s'ella diventasse femina del mondo voi diventerete sua messaggera col mondo et sareste da punir acerbamente, dove forse il fallo di lei non sembra del tutto incapace di scusa fondata sopra le nostre colpe [...], essortandovi di nuovo [...] a non uccider in un medesimo colpo l'anima e l'honor vostro insieme a quello della vostra figlia [...], non sostenete che non pur le carni della misera vostra figlia si squarcino et si vendano, ma d'esserne voi stessa il macellaio.?

En outre, dans un de ses testaments, elle prit la disposition de destiner une certaine somme d'argent à

due donzelle da bon, ma se si ritrovasse due meretrice che volessino lassar la cativa vita, e maritarsi, o monacharsi, in questo caso sii abrazado dette due meretrice, et non le donzelle. ${ }^{8}$

Ailleurs, elle avoue d'avoir des aspirations et des interêts élevés:

Io sono tanta vaga, e con tanto mio diletto converso con coloro che sanno per avere occasione ancora d'imparare, che, se la mia fortuna il comportasse, io farei tutta la mia vita e spenderei tutto 'l mio tempo dolcemente nell'accademie degli uomini virtuosi.' 
Dans une autre lettre, destinée à consoler un ami, elle lui rappelle en passant, ce qui est bien significatif, la chance qu'il avait d'être né homme et non pas femme comme elle. ${ }^{\mathrm{IO}}$ Les misères de la vie des courtisanes, - et pas seulement au XVI siècle -, ont été souvent évoquées. ${ }^{\text {I }}$ À Venise, une cortigiana onesta pouvait compter sur une vie peut-être moins amère qu'ailleurs, elle pouvait maintenir des relations plus ou moins libres avec les riches, les nobles et des personnages importants, accumuler des biens, participer à la vie littéraire et intellectuelle, publier. Toutefois, malgré tout cela, sa position est toujours restée incertaine: elle ne pouvait jamais être l'égale de ceux qu'elle fréquentait, être considérée comme une "femme comme il faut", ni compter sur un état stable. Au contraire, elle risquait d'être accusée d'immoralité, de " mauvaise vie » et pouvait s'attendre à des offenses, des vexations, des abus de toute sorte en tant que personne vivant dans un certain sens en marge de la société régulière. C'est contre ce statut faussé que Veronica Franco s'élevait. Pour défendre de façon audacieuse sa dignité, elle n'hésita pas de mettre à profit sa position particulière: ce qui était considéré comme honteux et qui était cause de marginalisation devint sous sa plume une source d'orgueil et de force.

Une telle intention se remarque indirectement dans les sonnets qu'elle adresse à Henri III, publiés six ans après son passage a Venise. En I580, Veronica Franco avait déjà connu l'amertume des fausses accusations et de la perte irréparable de ses biens. Peut-être désirait-elle se proposer de nouveau à l'attention de ses concitoyens, réparer les dommages provoqués par les événements plus récents, rappeler son statut d'antan, revenir aux temps écoulés. Les sonnets sont précédés par une dédicace plutôt conventionnelle, dans laquelle pourtant la belle Veronica ne manquait pas de rappeler à tout le monde que le roi était venu en personne dans son bumble domicile, qu'en partant il avait tenu à emporter avec lui en souvenir un portrait d'elle, en ce qu'elle appelle un échange, certes tout avantageux pour elle, un échange de faveurs et de sentiments, qu'il lui avait fait de gentilles et gracieuses propositions à propos du livre qu'elle avait l'intention - et le courage - de lui dédier, et qu'elle peut ainsi oser embrasser les genoux du souverain. Des motifs similaires ou apparentés reviennent ensuite de façon plus explicite dans les sonnets: le roi-Dieu, de sa propre volonté, se met au niveau de son bôtesse; il lui est reconnaissant pour la féminité naturelle qu'elle lui offre; entre eux deux naît un vif sentiment: 
Come talor dal ciel sotto umil tetto Giove tra noi qua giù benigno scende, e perchè occhio terren dall'alt'oggetto non resti vinto, umana forma prende;

così venne al mio povero ricetto, senza pompa real ch'abbaglia e splende, dal fato Enrico a tal dominio eletto, ch'un sol mondo nol cape e nol comprende.

Benché sì sconosciuto, anch'al mio core tal raggio impresse del divin suo merto, che 'n me s'estinse il natural vigore.

Di ch'ei, di tant'affetto non incerto, l'imagin mia di smalt'e di colore prese al partir con grat'animo aperto.

Prendi, re per virtù sommo e perfetto, quel che la mano a porgerti si stende: questo scolpito e colorato aspetto, in cui 'l mio vivo e natural s'intende.

E s'a essempio sì basso e sì imperfetto la tua vista beata non s'attende, risguarda a la cagion, non a l'effetto. Poca favilla ancor gran fiamma accende.

E come 'l tuo immortal divin valore, in armi e in pace a mille prove esperto, m'empìo l'alma di nobile stupore, così 'l desio, di donna in cor sofferto, d'alzarti sopra 'l ciel dal mondo fore, mira in quel mio sembiante espresso e certo. ${ }^{\mathrm{I}}$

Toutefois la voix de Veronica avait résonné de façon beaucoup plus énergique quelques années plus tôt, dans ses capitoli in terza rima, des pièces de longueur variable, de 39 à s65 vers, dans lesquelles elle affirmait déjà sa personnalité exceptionnelle. Dans les années I570, elle avait été attaquée par un auteur anonyme (que l'on identifie aujourd'bui avec Maffeo Venier), ${ }^{13}$ qui avait fait circuler à son propos des vers humiliants, offensants et assez vulgaires. La courtisane répondit à deux reprises, en attribuant injustement ces offenses à son amant, puis à un de ses cousins. À distance, ce n'est pas le résultat de la dispute ou 
de la tenson, qui est intéressant, mais le choix des arguments de la part de la poétesse et l'usage qu'elle en fait avec une rare habileté. Face à l'offense, elle réagit comme un homme, alors même qu'elle croit encore que l'offenseur est son amant; son courroux et son indignation sont si grands qu'elle le défie avec force, selon les habitudes des chevaliers, pour un combat sans pitié, et la stylisation maniériste de l'amplification poétique ne diminue en rien l'impact de son expression ni la nature de ses sentiments:

Non più parole: ai fatti, in campo, a l'armi, ch' io voglio, risoluta di morire, da sì grave molestia liberarmi.

Non so se 'l mio «cartel » si debba dire,

In quanto do risposta provocata:

Ma perchè in rissa de' nomi venire?

Se vuoi, da te mi chiamo disfidata;

$\mathrm{E}$, se non, ti disfido $[\ldots]$

$[\ldots]$

con queste proprie man, arditamente

ti trarrò fuor del petto il vivo core.

La falsa lingua, ch' in mio danno mente, Sterperò da radice [...]

Quest'è d'arditi cavalier buon uso. ${ }^{14}$

Veronica toutefois n'oublie pas son amour, car la haine, toute violente qu'elle est, n'a pas la force d'effacer la véritable passion amoureuse qu'elle a le courage d'affirmer; elle est donc prête à payer de sa propre vie la défense de sa dignité, et son discours souligne l'ambiguïté de l'effet final qui couronne la vengeance rêvée:

[...] pur disperata prenderò in diletto

D'esser al sangue in vendetta ricorsa:

Poi col coltel medesmo il proprio petto,

De la tua occision sazia e contenta,

Forse aprirò, pentita de l'effetto. ${ }^{\text {IS }}$

Cette ambiguïté pourtant n'est qu'une première étape, avant le changement d'allure du discours. Le combat mortel peut se transformer facile- 
ment en duel amoureux. En recourant à cette métonymie conventionnelle, la poétesse trouve l'occasion de souligner ouvertement, sans pruderie mais avec sûreté, le coté physique d'une relation que ne sanctionne pas la morale, la satisfaction qu'elle y trouvait et la nostalgie qu'elle en conserve:

Vuoi per campo il segreto albergo, quello.

Che de l'amare mie dolcezze tante

Mi fu ministro insidioso e fello?

Or mi si para il mio letto davante,

Ov'in grembo t'accolsi, e ch'ancor l'orme

Serba dei corpi in sen l'un l'altro stante.

[...] questo medesimo soggiorno,

Che fu de le mie gioie amato nido. ${ }^{16}$

Tout cela conduit à une conclusion audacieuse, où les images érotiques deviennent exceptionnellement explicites:

[...] ma se da te mi sia la pace offerta?

se la via prendi, l'armi poste in terra,

a le risse d'amor del letto aperta?

[...] Forse nel letto ancor ti seguirei,

e quivi teco guerreggiando stesa,

In alcun modo non ti cederei:

Per soverchiar la tua si indegna offesa

Ti verrei sopra, e nel contrasto ardita,

Scaldandoti ancor tu ne la difesa

Teco morrei d'egual colpo ferita. ${ }^{17}$

Veronica Franco a donc eu le courage de manifester avec force et habileté à la fois son orgueil et sa sexualité dans le capitolo destiné à son amant. Son indignation trouve une expression bien différente - et moins émotionnelle - dans la seconde réponse à la même offense, destinée à l'autre personnage qu'elle suspectait. Les arguments y sont beaucoup moins personnels, et la courtisane prétend prendre la parole au nom du sexe féminin en général:

e le donne a difender tutte tolgo

Contra di voi. ${ }^{18}$ 
Elle choisit comme point de départ le lieu commun de la faiblesse des femmes, que la vertu des hommes défend d'attaquer.

D’ardito cavalier non è prodezza [...]

[...] da cavalier non è, ch'abbia raccolta ne l'animo suo invitto alta virtute, e che a l'onor la mente abbia rivolta, con armi insidiose e non vedute, a chi più disarmato men sospetta dar gravi colpi di mortal ferute.

Men ch'agli altri ciò far poi se gli aspetta contra le donne, da natura fatte per l'uso che più d'altro a l'uom diletta: imbecilli di corpo, ed in nulla atte non pur a offender gli altri, ma se stesse dal difender col cor timido astratte. ${ }^{19}$

Les femmes pourtant ne sont pas condamnées à accepter cette condition et à rester toujours dans une position subalterne vis-à-vis des hommes. Veronica Franco parle de la "nature agile 》 des femmes, capables de s'adapter en toute circonstance, et elle réclame les droits de son sexe en rejetant fermement les conventions, quand elle déclare:

[...] vi mostrerò quanto al vostro prevaglia

Il sesso feminil [...]

Quando armate ed esperte ancor siam noi, render buon conto a ciascun uom potemo, ché mani e piedi e core avem qual voi; e se ben molli e delicate semo, ancor tal uom, ch'è delicato, è forte; e tal, ruvido ed aspro, è d'ardir scemo. Di ciò non se ne son le donne accorte; che se si risolvessero di farlo, con voi pugnar porìn fino a la morte $[. .$. Data è dal ciel la feminil bellezza, perch'ella sia felicitate in terra di qualunque uom conosce gentilezza. ${ }^{20}$

Elle n'hésite pas à proposer explicitement aux autres femmes son propre exemple, en soulignant la nécessité du courage: c'est seulement 
après s'être décidée à s'opposer à l'arrogance masculine, qu'elle a appris par expérience la valeur de son sexe:

[...] dal causato periglio animo presi, benché femina a molli opere nata; $\mathrm{e}$ in man col ferro a essercitarmi appresi, tanto ch'aver le donne agil natura, non men che l'uomo, in armeggiando intesi: perché 'n ciò posto ogni mia industria e cura, mercè del ciel, mi veggo giunta a tale, che più d'offese altrui non ho paura [...] Così nei casi avversi i savi fanno, che 'l lor utile espresso alfin cavare da quel, che nuoce da principio, sanno [...] $[\ldots .$.$] tra tante donne incominciar voglio io,$ porgendo essempio a lor di seguitarlo. ${ }^{21}$

En défiant son offenseur, la poétesse propose un combat littéraire et non pas érotique, laissant à son adversaire le choix des armes qu'elle connaît toutes: la lingua volgar venetiana, la toscana, la seria, la burlesca, la favella giornalmente usata, et dans lesquelles elle est également experte. ${ }^{22}$ Elle n'a pas peur non plus de lancer à la fin une attaque frontale:

E se ben «meretrice» mi chiamate, o volete inferir ch'io non vi sono, o che ve n'è tra tali di lodate.

Quanto le meretrici hanno di buono, quanto di grazioso e di gentile, esprime in me del parlar vostro il suono. ${ }^{23}$

Il est surprenant de voir avec quelle habileté Veronica Franco emploie le langage fondé sur les métaphores appartenant au champ du combat. ${ }^{24}$ Ses duels sont à la fois concrets et symboliques, avec des connotations qui nous transportent facilement, ayant touché le sens de la collision verbale, artistique ou intellectuelle, vers la sphère de l'érotique. Dans ce sens la poétesse renoue avec la tradition ou avec la mode du pétrarquisme, et en même temps elle s'y oppose de façon radicale, donnant un sens nouveau aux vieilles images: un sens à la fois matériellement et audacieusement sensuel et féminin. Encore plus significatif et intriguant de ce point de 
vue paraît son dialogue avec l'amant, Marco Venier, qui ouvre les Rime: le jeu avec le pétrarquisme et l'anti-pétrarquisme, le refus d'une liaison purement poétique et verbale en faveur d'un amour "vrai», fondé sur les faits et non pas sur les paroles, une consciente mise en relief des propres qualités, des propres préférences et des goûts dans cette liaison, la promesse ouverte des plaisirs charnels font $d u$ capitolo de Veronica Franco un curieux mélange d'art, de coquetterie et d'autopromotion érotique, voire même de proxénétisme, de ruffianeria ouverte, où elle réussit avec une rare virtuosité de se mettre en valeur elle-même, peut-être pour se vendre mieux. ${ }^{25}$

Les mêmes motifs apparaissent en d'autres lieux encore. Si les femmes sont faibles, ce n'est qu'en face de l'amour, et la vraie cause en est que le genre humain, à la différence de tous les autres êtres vivants, n'est pas capable de vivre ce sentiment avec sérénité. Il n'est pas sans importance de noter que le discours s'inscrit dans des plaintes adressées à un amant lointain et indifférent, et que les restrictions imposées aux femmes ne permettent aucune liberté et ne pardonnent aucune faute:

[...] e l'uom, dal cielo a dominar eletto

tutti gli altri animali de la terra,

dotato di ragione e d'intelletto;

l'uom che, se non vuol, rado o mai non erra,

fa, nei desir d'amor dolci, a se stesso

così continua abominosa guerra,

sì ch'a lui poi d'amar non è concesso, senza trovar di repugnanti voglie

de la persona amata il core impresso.

[...] Far non può de le donne resistenza

la natura si molle ed imbecilla,

di Venere del figlio a la potenza;

picciol aura conturba la tranquilla

feminil mente; e di tepido foco

l'alma semplice nostra arde e sfavilla.

E quanto avem di libertà più poco, tanto 'l cieco desir, che ne desvia, di penetrarne al cor ritrova loco: sì che ne muor la donna, o fuor di via esce de la comun nostra strettezza, e per picciolo error forte travia. ${ }^{26}$ 
Ailleurs, elle rappelle la tâche qu'elle a assumée, défendre toutes les femmes:
Povero sesso, con fortuna ria
sempre prodotto, perch'ognor soggetto
e senza libertà sempre si stia!:
Né però di noi fu certo il diffetto,
che se ben come l'uom non sem forzute, come l'uom mente avemo ed intelletto.
Né in forza corporal sta la virtute, ma nel vigor de l'alma e de l'ingegno [...] e certa son che in ciò loco men degno non han le donne, ma d' esser maggiori degli uomini dato hanno più d'un segno. [...] e la donna, perchè non segua il male, s'accomoda e sostien d'esser vassalla. Ché se mostrar volesse quanto vale in quanto a la ragion, de l'uom sarìa di gran lunga maggiore, e non che eguale. ${ }^{27}$

Mais elle souligne encore, non sans quelque ironie:

Per non guastar il mondo, ch'è si bello per la specie di noi, la donna tace, e si sommette a l'uom tiranno e fello, che poi del regnar tanto si compiace, sì come fanno 'l più quei che non sanno (ché ' 1 mondan peso a chi più sa più spiace). ${ }^{28}$

Des études récentes sur la production littéraire « au féminin » à l'époque de la Renaissance en Italie soulignent d'une part les efforts et les tentatives des auteurs femmes pour exprimer dans un langage nouveau la conscience de leur propre identité féminine et leur sexualité, et d'autre, elles mettent en relief le fait que plusieurs compositions signées par ces auteurs se situent bien dans les schémas idéologiques que le monde masculin avait imposés aux femmes. ${ }^{29}$ Veronica Franco semble unir les deux tendances: elle déclare accepter son rôle de femme objet/symbole/ agent sexuel, voire même d'y trouver des satisfactions, et elle se montre capable de le défendre et de le manifester ouvertement sans bonte. 
Toutefois, même si son cuvre ne présentait aucune menace sérieuse pour l'idéologie de la chasteté féminine et de l'autorité des hommes, elle réussit tout de même à esquisser dans ses poésies une solution alternative, en donnant une image de femme cultivée et intellectuellement brillante, consciente de soi, déplorant la soumission de son sexe qu'elle trouve injuste et contre laquelle elle ose protester en contestant de front l'idée de l'infériorité des femmes, se présentant comme une femme acceptant sa sexualité, capable d'en parler et d'y réclamer un rôle actif..$^{30}$ Il est vrai que ses rime sont adressées à différents hommes: mais en réalité, visent-elles uniquement un public masculin? Tout ce qu'elle dit se laisset-il si facilement circonscrire à son statut de cortigiana entièrement marginalisée, fût-il de cortigiana onesta, par rapport aux femmes véritablement honnêtes? Il semble bien qu'elle avait à transmettre des messages différents, destinés à des lecteurs appartenant à des publics différents, et qu'elle savait jouer avec eux.

Mais sa bravoure, son assurance, son aplomb, voire même peut-être une certaine arrogance, on ne saurait jusqu'à quel point simulée, se seront fort apaisés quelques année plus tard. C'est que les expériences douloureuses qui ont marqué sa vie dans la deuxième moitié des années I570 lui ont fait voir la beauté comme une marchandise, quand elle évoque:

la vanità di biondeggiarsi et del lisciarsi, [...] capegli inanellati d'intorno alla fronte e 'l collo, col petto spalancato che esce fuor dei panni, con la fronte alta e scoperta, con tutti quegl'altri abbellimenti che s'usano di fare perché la mercantia trovi concorrenza nello spedire $\left[\ldots . . .^{31}\right.$

La condition de courtisane n'est pas seulement difficile:

[...] la profession delle cortegiane nella quale si ha gran fatica di riuscire chi sia bella et habbia maniere et giudizio et conoscenza di molte virtù.

Elle est encore dangereuse, et Veronica en parle avec connaissance précise et détaillée:

[...] in ogni essito misera et troppo infelice: troppo contrario al senso humano è l'obligar il corpo e l'industria di una tal servitù che spaventa solamente a pensarne, darsi in preda di tanti con rischio di essere dispogliata, d'esser rubata, d'esser uccisa, ch'un solo dì ti toglie quanto con molti in molto tempo hai acquistato, con tant'altri pericoli d'ingiurie et 
d'infermità contagiose et spaventose. Credete a me, fra tutte le sciagure mondane questa è l'estrema. ${ }^{32}$

Avec cela, Veronica Franco entre dans un nouveau rôle, celui de conseillère et de consolatrice, qui souvent moralise. Des accents ayant trait à cette vocation se laissaient déjà voir déjà dans les capitoli publiés auparavant, dans de fréquentes remarques de caractère général, dispersées çà et là, à propos des adversités et des troubles apportés par la vie et l'amour, auxquels il faut faire face avec force et patience. La sagesse, une sagesse bien consciente de soi et dûment manifestée, remplace alors la sensualité débordante, sans diminuer en rien le rôle de Véronique en tant que protagoniste de sa vie. Plusieurs lettres mettent cet aspect en premier lieu. Elle veut réconforter:

poiché $\mathrm{i}$ travagli dell'animo non si purgano con beveraggi medicinali, ne al batter del polso si conoscono l'indisposizione dell'angoscioso pensiero;

alle parole che usaste meco ragionando l'altra sera m'accorsi di un animo in voi turbato et mal composto per la vostra contraria fortuna;

prima che 'l tempo, vulgar medico, e da dozzina, risani la piaga del vostro dolore. $^{33}$

Ou bien, elle se prononce sur «le monde»:

Nel governo della famiglia nuoce la molta severità non meno che la troppa indulgentia;

Le cose del mondo che non sono ordinate sotto perpetua legge simile all'invariabile natura dei movimenti celesti, sono disposte in tal maniera;

S'el dolor dell'animo bastasse a ripararci ai gravi colpi del mondo et della fortuna, in quei casi ancora che sono capaci di remedio, gran prudentia sarebbe lasciando da parte tutte l'altre cose, per ristoro dell'avversità viver in doglia e in pianto continuo mettendo sopra i termini d'ogni allegrezza et d'ogni riso il duolo e le lagrime, ma perche l'affanno della mente aggiunto alle percosse della nemica sorte tanto piu aggrava l'animo delle miserie. ${ }^{34}$ 
À un jeune débutant, elle donne une leçon de sagesse en amour, tout en soulignant sa vocation aux études et à la culture dont elle donne des preuves incontestables dans d'autres lettres farcies d'érudition:

tra tutti coloro che pretendono di poter insinuarsi nel mio amore a me sono estremamente cari quei che s'affaticano nell'essercizio delle discipline e delle arti ingenue delle quali (se ben donna di poco sapere, rispetto massimamente alla mia inclinazione e al mio desiderio) io sono tanto vaga e con tanto mio diletto converso con coloro che sanno per haver occasione ancora d'imparare [...].

Elle insiste aussi sur la responsabilité morale de la femme, dévoilant à cette occasion un autre aspect de l'amour, qui n'est qu'un

incitamento in noi il qual secondo che vien moderato nel nostro affetto e cagion di cose contrarie, si che dove altri trasportato dalla temerità del senso e incorso nel proprio suo danno et nella sua espressa vergogna, non e mancato chi frenandosi dalle cose dishoneste per non dispiacer alla sua donna et applicando la mente alla virtù per meritar la grazia sua ha fatto imprese illustri e memorabili.35

Tandis que rien ne suggère que pour Véronique les deux aspects de l'amour, charnel et spirituel, s'excluraient nécessairement au lieu de cohabiter pacifiquement, le sens de la dignité chez la courtisane-poétesse trouve encore un point de référence: sa venezianità, où la foi joue un rôle important. ${ }^{36}$ À plusieurs reprises, elle revient sur l'image glorieuse de sa ville natale:

[...] meraviglia e stupor de la natura.

Questa dominatrice alta del mare, regal vergine pura, inviolata, nel mondo senza essempio e senza pare [...]

e dove anch'io, la Dio mercè, son nata; [...] fabricata è Vinegia sopra l'acque, per sopranaturai celeste aviso:

in questa il Re del ciel si compiacque di fondar il sicuro, eterno nido de la sua fè, ch'altrove oppressa giacque; e pose a suo diletto in questo lido tutto quel bel, tutta quella dolcezza. ${ }^{37}$ 
Les mêmes images reviennent encore:

[...] del mar reina, in mezzo 'l mar assisa, a' cui piè l'acqua giunta umile cade, e per diverso e tortüoso calle s'insinua a lei per infinite strade. [...] sì che nel tempo novo o ne l'antico non fu mai chi tentasse violarla, ch'al pensar sol confuse ogni nemico. Tutto 'l mondo concorre a contemplarla, come miracol unico in natura, più bella a chi si ferma più a mirarla $[\ldots ..] .^{38}$

Dans une de ses lettres consolatoires, elle rappelle à son destinataire, un dernier avantage de sa situation personnelle, parmi tous les autres:

Tra gli huomini di diversi paesi v'habbia dato per patria una Città non barbara, non serva ma gentile et non pur libera ma signora del mare et delle piu belle parti dell'Europa, città veramente donzella immacolata et inviolata, senza macchia d'ingiustizia et non mai offesa in se stessa da forza nemica, per incendio di guerra, ne per combustione del mondo, in ogni revolutione sola per miracolo conservata non pur intiera ma ne ancora tentata da contrario impeto, si come sola per miracolo fondata nel mezzo dell'acque e con meravigliosa tranquillità stata in piedi e sempre aumentata per infinito spatio di tempo, Città piena di meraviglia et di stupore tale che per descriversi senza vedersi non si conosce ne vien compresa da intelletto umano. ${ }^{39}$

Veronica Franco assume ainsi toujours le rôle de protagoniste, de sa vie comme de sa poésie, et elle réclame toujours l'attention et le respect. Quelle soit la situation où elle se trouve, elle n'hésite pas à se prononcer avec une respectable sûreté. Vise-t-elle la compensation des chagrins que la vie réelle ne lui avait pas épargnés? Quoi qu'il en soit, elle ne se replie pas sur elle-même, mais elle laisse ses interlocuteurs - et ses lecteurs croire qu'ils ont devant eux une personnalité forte, intègre, si l'on peut dire, et digne de considération. La "nemica sorte», la fortune adverse, ne lui a pas laissé du temps pour continuer son cuvre. Morte âgée de 45 ans, Veronica Franco reste toujours un des personnages qui ont fortement marqué le passé brillant de la Serenissima. 
La rencontre de la poétesse-courtisane et du roi n'a été qu'un épisode éphémère et sans conséquences. Il reste à notre imagination de se représenter les chances auxquelles pouvait songer cette femme ambitieuse et mal à l'aise dans le corset étroit des convenances. Et les chances, l'avenir auxquels pouvait penser le jeune monarque, profondément déçu par une expérience polonaise qui avait enfin pris fin, qui revenait de son exil "doré» vers un monde qu'il préférait et qui saluait son retour en lui offrant ce qu'il avait de mieux. Pour tous les deux l'avenir s'est montré bien dur et bien éloigné des rêveries poétiques.

Piotr Salwa 
I. Voir Rocco Benedetti, Le Feste et Trionfi fatti dalla Serenissima Signoria di Venetia nella Felice Venuta di Henrico III, Venezia, I 574. L'ouvrage a été réimprimé la même année par plusieurs éditeurs; il existe une traduction française publiée sous le titre de Discours des triomphes et resjouissances faicts par la Serenissime Seigneurie de Venise à l'entrée heureuse de Henry de Valois, Lyon, M. Jouve, i 574.

2. «Le Lundi à souper, 7 de Novembre, la Signora Veronica Franco, gentifemme Venitienne, envoya vers luy pour lui presenter un livre de Lettres qu'elle a composé; il fit donner deux escus audict homme », Michel de Montaigne, Journal de voyage, éd. F. Rigolot, Paris, Presses universitaires de France, I 992, p. 68. Son nom avec l'adresse et l'indication du prix qu'elle demandait aux clients (qui n'était pas des plus élevés, ce qui a suscité des doutes de la part des historiens) se trouvait dans le célèbre Catalogo de tutte le principal et più honorate cortigiane di Venetia (ca. I 56 ). Voir F. Rigolot, « Montaigne et Veronica Franco: de la courtisane à la femme de lettres », Montaigne Studies, vol. I 5, 2003, p. I I7-I 30.

3. Margaret F. Rosenthal, The Honest Courtesan: Veronica Franco, Citizen and Writer in Sixteenth-Century Venice, Chicago, University of Chicago Press, i 992; Dangerous Beauty, directed by Marshall Herskovitz, Warner Bros. et $20^{\text {th }}$ Century Fox, 1998. Pour la biographie, on renverra à la notice "Veronica Franco» par Floriana Calitti, in Dizionario Biografico degli Italiani, vol. 50, Roma, Istituto dell'Enciclopedia Italiana, i 998, ainsi qu'à Veronica Franco, Poems and Selected Letters, edited and translated by Ann Rosalind Jones and Margaret F. Rosenthal, Chicago, The University of Chicago Press ("The Other Voice in Early Modern Europe"), 1998.

4. Voir Michael Asimow (UCLA Law School), Dangerous Beanty: A Trial of a Courtesan, May I998, at http://usf.usfca.edu/pj/articles/dangerousbeauty.htm.

5. Terze rime di Veronica Franca al serenissimo signor duca di Mantoua et di Monferrato, [Venezia ?], [s.n.], [ 1575 ?]; Lettere familiari a diversi della s. Veronica Franca all'illustriss. et reverendiss. Monsig. Luigi d'Este cardinale, [Venetia?], [s.n.], [I 580 ?].

6. Rime di diversi eccellentissimi auttori nella morte dellillustre sign. Estor Martinengo conte di Malpaga. Raccolte et mandate allillustre et valoroso colonnello il s. Francesco Martinengo, suo fratello, conte di Malpaga dalla signora Veronica Franca, [Venezia ?], [s.n.], [ I 575 ?]. Voir Deanna Shemek, "The Collector's Cabinet. Lodovico Domenichi's Gallery of Women ", in Strong Voices, Weak. History. Early Women Writers and Canons in England, France and Italy, edited by Pamela Joseph Benson and Victoria Kirkham, Ann Arbor, The University of Michigan Press, 2005, p. 239-262.

7. Lettere familiari a diversi, cit., p. 4I-42, 43, 45: «Sa ruine ne peut pas être séparée de la vôtre, parce que vous êtes la mère et parce que si elle devenait femme du monde, vous deviendriez sa messagère avec le monde, et vous seriez à punir sévèrement, tandis que sa faute ne semble peut-être pas impossible à être excusée, vus nos propres défauts [...], vous tueriez d'un seul coup votre âme et votre honneur ainsi que ceux de votre fille [...], ne permettez pas que non seulement la chair de votre pauvre fille soit déchirée et vendue, mais que vous-même en soyez le boucher» (nous traduisons). 
8. Voir Margaret F. Rosenthal, The Honest Courtesan. Veronica Franco, op. cit., p. i 3 -II5: «Deux demoiselles de bien, mais si l'on trouvait deux catins qui voudraient laisser la mauvaise vie et se marier ou entrer dans un couvent, dans ce cas-là on prendra les deux catins et non les demoiselles ». Voir également Ann Rosalind Jones, «Bad Press. Modern Editors vs. Early Modern Women Poets (Tullia d'Aragona, Gaspara Stampa, Veronica Franco)», in Strong Voices, Weak History. Op. cit., p. 303 et suivantes.

9. Lettere familiari a diversi, cit., p. 28-29: «Je suis tellement désireuse et avec un tel plaisir je converse avec ceux qui savent pour avoir encore l'occasion d'apprendre que si mon sort le permettait, je passerais toute ma vie et tout mon temps agréablement dans les académies des homme vertueux ».

ı०. "V'habbia attribuito il sesso del maschio et non si come a me quel della femmina ", Lettere familiari a diversi, cit., p. 6.

I I. Voir Paul Larivaille, La vie quotidienne des courtisanes en Italie au temps de la Renaissance, Paris, Hachette, 1975.

i 2. Je traduis d'après Veronica Franco, Rime, a cura di Stefano Bianchi, Milano, Mursia, I 995. I. "Comme parfois du ciel sous un humble toit descend entre nous le bienveillant Jupiter, et pour que l'œil terrestre ne reste pas abattu par ce haut objet, il prend la forme humaine, ainsi vint dans mon pauvre domicile, sans pompe royale qui éblouit et resplendit, Henri, élu par la fortune au royaume qui ne se limite pas à un seul monde. Et bien qu'ainsi déguisé, il a imprimé aussi dans mon cœur un tel signe de ses mérites divins que ma vigueur naturelle s'est éteinte. Et lui, non pas inconscient de cet effet, en partant il a pris avec lui avec gratitude et esprit ouvert mon image en email et en couleur. » II. « Roi suprême et parfait, prend ce que la main s'étend pour te donner, cette image gravée en couleurs d'où l'on peut comprendre mon aspect vivant et naturel. Et si à un objet si bas et si imparfait ton œil divin ne s'arrête pas, regarde la cause et non pas l'effet, une petite étincelle peut provoquer une grande flamme. Et ainsi que ton courage divin et immortel, expérimenté dans la guerre et dans la paix par mille épreuves, a rempli mon âme de noble étonnement, regarde dans mon image l'expression certaine du désir, souffert dans le cœur d'une femme, de t'élever au dessus du ciel».

I3. A propos de la polémique avec ce personnage, voir Dolora Chapelle Wojciehowski, «Veronica Franco vs. Maffio Venier: Sex, Death, and Poetry in Cinquecento Venice », Italica, vol. 83, no. 3/4 (Fall - Winter, 2006), p. 367-390.

i4. Veronica Franco, Rime, Capitolo XIII, i-8; i7-20; 58: «Plus de mots, aux faits, dans le champ, aux armes parce que décidée à mourir, je veux me libérer d'un grand ennui. Je ne sais pas si le mien doit s'appeler un défi, car je donne une réponse provoquée en avance - mais pourquoi discuter des mots? Si tu veux je m’appelle défiée par toi, si non, c'est moi qui te défie. [...] Avec mes propres mains, avec courage, je te tirerais le cœur vif hors de la poitrine. [...] Ta fausse langue qui ment à mon tort, je l'extirperai de sa racine [...] Telle est l'habitude des chevaliers courageux $[\ldots] »$. 
i 5. Veronica Franco, Rime, 23-27: "Même désespérée je trouverai du plaisir en me vengeant jusqu'à verser du sang, et peut-être après, regrettant l'effet, j'ouvrirai avec le même couteau ma propre poitrine, satisfaite et contente de t’avoir tué ».

i6. Veronica Franco, Rime, 3 I-36; 40-4i: «Est-ce que tu veux pour le champ le secret lieu qui a m'avait octroyé - insidieux et traitre - des nombreux plaisirs or si amers? Or j'ai devant les yeux mon lit dans lequel je t'ai accueilli dans mon sein et où les traces de nos corps embrassés restent encore. [...] ce même lieu qui a été le nid bien aimé de mes joies ».

17. Veronica Franco, Rime, 70-72; 79-85: «Et si tu m’offrais la paix? [...] Si après avoir déposé les armes tu prends la voie ouverte aux dispute du lit?[...] Peut-être je te suivrais au lit et couchée en guerroyant je ne te céderais pas.[...] Pour surmonter ton indigne offense, je viendrais au-dessus de toi et courageuse dans le combat, toi te réchauffant dans la défense, je mourrais avec toi blessée du même coup ».

i 8. Veronica Franco, Rime, Capitolo XVI, 79-80: «J'ai l'intention de défendre toutes les femmes contre vous ».

i 9. Veronica Franco, Rime, i-i 5: «Ce n'est pas une prouesse digne d'un chevalier hardi - ce n'est pas digne d'un chevalier qui abrite dans son âme invaincue la haute vertu et qui tourne son esprit à l'honneur - de donner des graves coups et des blessures mortelles avec des armes insidieuses et inattendues à qui, désarmé, moins le soupçonne. Moins qu'aux autres, on s'attend que l'on le fasse aux femmes, faites par la nature pour ce qui plait aux hommes plus que toute autre chose, faibles du corps et non seulement incapables d'offenser les autres, mais inhabiles même à se défendre elles-mêmes avec leur cœur timide ».

20. Veronica Franco, Rime, 94-95; 64-72; 84-86: «Je vais vous montrer combien le sexe féminin soit supérieur au vôtre [...] Quand nous sommes armées et expertes, non sommes capables de rendre bon compte à chaque homme, car nous avons les mains, les pieds et le cœur comme vous, et si nous sommes tendres et délicates, il y des hommes qui sont délicats et forts, et d'autres qui sont rêches et rudes et néanmoins manquent de courage. De tout cela les femmes ne se sont pas encore rendu compte, car si elles s’y étaient décidées, elles pourraient combattre avec vous jusqu'à la mort [...] La beauté des femmes est donnée par le ciel, pour qu'elle soit le bonheur de chaque homme qui sait ce que c'est la gentillesse».

2i. Veronica Franco, Rime, 32-39; 43-45; 74-75: «Du danger où je me suis trouvée j’ai pris le courage et bien que je sois femme née pour les tendresses, j’ai commencé à m'exercer avec le fer à la main, si agile est la nature des femmes capables de combattre non moins que les hommes. Et parce que j'y ai mis tout mon esprit et tous mes soins, grâce au ciel je me vois arrivée au point où je n'ai pas peur des offenses des autres. [...] De cette manière se comportent les sages qui savent tirer à la fin leur profit de ce qui nuit au début. [...] Parmi tant de femmes je veux commencer et donner l'exemple aux autres de me suivre».

22. Veronica Franco, Rime, Capitolo CVI, i i 2 et suiv. 
23. Veronica Franco, Rime, Capitolo XVI, i 78 - i 83 : «Et quoique vous m’appelez catin, ou bien vous voulez dire que je ne le suis pas, ou bien vous laissez croire que parmi elles il y en a qui méritent l'éloge. En s'adressant à moi, votre langage exprime combien les catins ont de bon, de charmant et de gentil ».

24. Sur les questions stylistiques et rhétoriques liées à la littérature féminine, voir Fabio Finotti, «Women Writers in Renaissance Italy. Courtly Origins of New Literary Canons », in Strong Voices, Weak History.... Op. cit, p. I 2 I-I 45.

25. Il me semble toutefois exagéré voir dans les rime de Veronica Franco de la pornographie; voir à ce propos Giovanna Rabitti, Lyric Poetry, I500-I650, in $A$ History of Women's Writing in Italy, edited by Letizia Panizza and Sharon Wood, Cambridge, Cambridge University Press, 2000, p. 47.

26. Veronica Franco, Rime, Capitolo XXII, 6i-69; 73-84: « et l'homme, élu par le ciel pour dominer tous les autres animaux de la terre, doué de raison et d'entendement, l'homme qui ne se méprend que rarement ou pas du tout, s'il ne le veut pas, il fait - dans son désir des douces amours - une telle guerre continuelle et abominable à soi-même qu'il ne peut pas aimer sans trouver chez la personne qu'il aime des désirs contraires. [...] La nature tendre et faible des femmes ne peut pas résister à la puissance du fils de Vénus, un souffle léger trouble leur esprit tranquille et même un feu tiède fait brûler et s'enflammer leur âme simple. Et quand nous avons un peu plus de liberté, aussitôt le désir aveugle qui fait perdre le droit chemin réussit à pénétrer dans notre cœur, de façon que la femme en meurt ou bien s'éloigne des règles qui nous sont communes, et par une légère faute fortement s'égare ».

27. Veronica Franco, Rime, Capitolo XXIV, 55-66; 80-84: « Pauvre sexe qui naît toujours avec la fortune contraire, parce qu'à chaque instant il se trouve dominé et sans liberté. Certainement ce n'est pas par notre faute, parce que même si nous ne sommes fortes comme les hommes, nous avons l'esprit et la raison comme eux, et la vertu ne réside pas dans la force du corps mais dans la vigueur de l'âme et de l'entendement. Quant à cela, je suis sure que les femmes ne sont pas inférieures aux hommes, mais au contraire, elles ont donné plusieurs preuves d'être supérieures à eux. [...] Une femme s'adapte pour empêcher le mal et elle consent d'être subordonnée, mais si elle voulait démontrer combien elle vaut quant à l'esprit, elle dépasserait l'homme de loin, loin de lui être égale».

28. Veronica Franco, Rime, 88-93: « Pour ne pas gâcher le monde qui est si beau par notre espèce, la femme se tait et se soumets à l'homme tyran et traître lequel se plait ensuite de régner dans le monde comme le font surtout ceux qui savent peu (vu que le fardeau mondain ne plait pas à ceux qui en savent plus)».

29. A ce sujet, voir par exemple Virginia Cox, "Women Writers and the Canon in Sixteenth-Century Italy", in Strong Voices, Weak History...., Op. cit.,pp. I4-3 I (avec une riche bibliographie), ainsi que Deanna Shemek, "The Collector's Cabinet. Lodovico Domenichi's Gallery of Women », ibid., p. 239-262.

30. Malgré les débats de l'époque, sur lesquels il existe aujourd'hui une vaste bibliographie, la persistance des stéréotypes ne semble pas sérieusement mena- 
cée. A titre d'exemple on pourrait rappeler le recueil de lieux communs composé et publié par Lodovico Guicciardini, destiné à un public cultivé et à la mode à son temps, où l'auteur affirme que c'est vraiment une malchance de naitre femme dans ce monde, et cela par plusieurs raisons, en premier lieux parce que celles qui sont célèbres ou dont le nom est connu sont très rares, que non seulement elles ne donnent pas de succession à leur maison, mais qu'en se mariant elles ne conservent même pas leur nom, qu'elles sont faibles de forces, d'esprit et d'entendement, inaptes aux actes ou exercices d'importance, soumises aux hommes, soumises à la nature et à différentes lois et coutumes auxquelles l'homme n'est pas soumis, Lodovico Guicciardini, L'ore di ricreazione, a cura di Anne-Marie van Passen, Leuven-Roma, Leuven University Press/Bulzoni editore, I990, $\mathrm{n}^{\circ} 58$, p. $65-66$ (notre traduction).

3 I. Lettere familiari a diversi, cit., p. 42-43: «la vanité de blondir les cheveux et de lisser la peau [...] avec les cheveux en boucles autour du front et du cou, avec la poitrine exposée qui sort des vêtements, avec le front haut et découvert, avec tous les autres ornements que l'on a l'habitude de mettre pour que la marchandise se vende plus rapidement».

32. Lettere familiari a diversi, cit., pp. 44-45: «la profession des courtisanes dans laquelle il est difficile de réussir même pour celle qui soit belle, ait de bonnes manières et du jugement, et qui connaisse plusieurs vertus; [...] dans tous ses effets misérable et malheureuse: il est trop contraire au sens humain d'obliger le corps et l'esprit à un tel esclavage qui effraie déjà quand on y pense seulement, de se donner à tant d'hommes avec le risque d'être dépouillée, dérobée, tuée, parce qu'un seul t'enlève tout ce que tu as acquis pendant de longues années, avec tant d'autres dangers d'offenses ou de maladies contagieuses et épouvantables. Croyez-moi, de tous les malheurs mondains, celui-ci est extrême ».

33. Lettere familiari a diversi, cit., pp. 3, 4, I 3: «vu que des troubles de l'âme on ne se débarrasse pas avec des breuvages médicaux, et que le rythme du pouls ne permet pas de connaître la mauvaise disposition des pensées angoissées; [...] l'autre soir je me suis rendu compte, grâce aux mots que vous avez prononcés parlant avec moi, que votre esprit est troublé et mal composé à cause de votre fortune contraire; [...] avant que le temps - médecin vulgaire et médiocre guérisse la plaie de votre douleur».

34. Lettere familiari a diversi, cit., pp. 23, 26, 54: «dans la direction d'une famille trop de sévérité nuit autant que trop d'indulgence; [...] les choses du monde qui ne sont pas soumises aux lois perpétuelles semblables à la nature invariable des mouvements du ciel, sont disposée de cette manière; [...] si la douleur de l'âme était suffisant à nous protéger contre de graves coups du monde et de la fortune, dans ces cas où il y a encore un remède, il serait bien prudent de laisser de coté toutes les autres choses et vivre en douleur et en larmes continuelles, pour se restaurer de l'adversité, préférant le deuil et les pleures à toute joie et au rire, mais étant donné que les soucis de l'esprit, ajoutés aux coups de la fortune contraire, rendent l'âme encore plus affligée par les misères ».

35. Lettere familiari a diversi, cit., pp. 28, 27: «Parmi tous les hommes qui prétendent de pouvoir s'insinuer dans mon amour, ceux qui me sont extrêmement 
chers se donnent à l'exercice des disciplines et des arts de l'esprit dont je suis tellement désireuse (quoique je sois une femme peu cultivée, surtout par rapport à mes inclinations et à mes désirs) et avec un tel plaisir je converse avec ceux qui savent pour avoir encore l'occasion d'apprendre [...]; incitation en nous, laquelle, selon si elle est modérée dans notre affection, peut être cause des choses contraires, ainsi que là où les uns entrainés par la témérité des sens sont encourus dans leur propre dommage et dans leur honte explicite, il y en a eu qui se sont freinés devant les choses déshonnêtes pour ne pas déplaire à leurs dames et tournant leur esprit à la vertu pour mériter leur faveurs ont fait des choses illustres et mémorables ».

36. La venezianità de Véronica Franco a naturellement des limites; elle compare à plusieurs reprises le peuple vénitien et ses coutumes à la lie, Letter familiari a diversi, cit., p. 7.

37. Veronica Franco, Rime, Capitolo XII, 2 I-27; 38-44: «merveille et stupéfaction de la nature, haute dominatrice de la mer, vierge royale pure et inviolée, sans égale et sans autre exemple dans le monde [...], où grâce au ciel je suis née, moi aussi [...], Venise est construite sur l'eau par un ordre surnaturel du ciel, en elle le roi céleste s'est plu à fonder un nid éternel et sûr de sa foi qui est oppressée ailleurs, et pour son plaisir il a posé sur ce rivage toute cette beauté et toute cette douceur».

38. Veronica Franco, Rime, Capitolo XXII, I62-165; i69-I74: «La reine de la mer, posée au milieu des eaux, aux pieds de la quelle l'eau arrive et pénètre dans ses nombreuses et tortueuses ruelles par des manières infinies [...] Dans les temps récents ou dans les temps anciens personne n'a jamais tenté de la violer, car seulement à y penser tout ennemi se confond. Tout le monde accourt à la contempler comme unique merveille de nature, elle parait plus belle à qui plus s'arrête à la regarder».

39. Lettere familiari a diversi, cit., p. 6-7. «Parmi les hommes provenant des différents pays vous avez pour patrie une ville non barbare, non assujettie, mais gentille et non seulement libre mais souveraine de la mer et des plus belles parties de l'Europe. Une ville qui est vraiment une demoiselle immaculée et inviolée, sans l'ombre d'injustice, jamais offensée par des forces hostiles, par une incendie de guerre ou casuelle, à chaque perturbation conservée par miracle, non seulement entière mais jamais tentée par des forces adverses, par miracle fondée au milieu des eaux et avec une merveilleuse calme restée debout, toujours grandissant par un temps infini. Ville pleine de merveille et étonnante, que l'esprit humain ne peut pas comprendre ni connaitre par les seules descriptions sans l'avoir vue ». 